\title{
Multimedia Teaching Platform Construction for Fashion Design Based on Simulation and Synchronous Teaching System
}

\author{
https://doi.org/10.3991/ijet.v13i05.8438 \\ Peng Qu \\ University of Science and Technology Liaoning, Anshan, Liaoning \\ 3121383141 @qq. com
}

\begin{abstract}
Service Oriented Architecture (SOA) technology can connect different service functions of application program through the well-defined interface and then form a sound distributed system through proper control process. SOA-based simulation and synchronous teaching system can give play to agility and high efficiency of this technology, so its practical value is high. Fashion design and other design majors have high requirements for multimedia software. The good presentation effect has great significance for design major teaching. Based on analyzing current situation of computer aided technology and fashion design teaching, theory construction of SOA-based simulation and synchronous teaching system was first conducted in this paper. Then, multimedia platform for simulation and synchronous teaching system was designed according to the specific requirements of fashion design teaching. Finally, the platform effect was tested. This paper verified that, SOA-based simulation and synchronous teaching system has good application effect in Fashion Design course and college teaching of design major.
\end{abstract}

Keywords-SOA distributed software, simulation and synchronous teaching system, fashion design, multimedia

\section{Introduction}

Computer aided technology is a theory and method based on computer activity in the specific field, including aided design, aided manufacturing, aided teaching and aided drawing, etc. aid mainly stresses the leading role of human. A man-machine interactive system forms through human coordination. To be specific, computer aided design conducts product design through computer system so as to reach the better design effect as far as possible. It is mainly applied in machine manufacturing and architectural composition. Computer aided instruction adopts the computer as the medium for teaching, drives education method changed and then improves teaching quality. In recent years, SOA distributed software has gained rapid development. The maturity of this technology provides the possibility for computer aided technology to go deep into each field. 
SOA is service-oriented architecture. It is a component model and can link the defined interface with different function units of application program [1]. In the architecture. The interface exists in a neutral form and is independent of hardware platform and system. Different commuter systems can interact through SOA. With SOA model, people can construct distributed software including multiple sub-systems so as to meet multiple needs of design teaching. By applying SOA-based simulation and synchronous teaching system, designers can have a better understanding of work depth and width and try more type of color and style collocation so as to gain the best design effect. Through computer-aided software simulation, designers can effectively control the whole effect of works and clearly display design thought and design idea [2].

The rapid development of computer aided technology brings the huge change to college fashion design teaching. The original plain and manual teaching gradually tends to digitization and automation [3]. CAD and Photoshop have played an important role in college design teaching. On one hand, they can enrich design expression methods; on the other hand, they can enhance teaching explanation of design works. The introduction of computer aided technology greatly improves teaching efficiency and reduces manual deviation. Based on the previous researches on computer-aided software, this paper mainly studied the design of SOA-based simulation and synchronous teaching system and investigated its application effect by taking fashion design major as the object to offer support for further promotion of computeraided software in college design teaching.

\section{State of the art}

In the background of modern education, the requirements demand of design courses represented by fashion design for advanced technology and optimized learning environment is more urgent. People hope to transform computer aided technology and apply it in practical teaching. In recent years, the design of SOA-based distributed software has gradually become research hotspot of domestic and overseas scholars and attracted numerous experts and scholars from the fields of computer science and education. The researches of most scholars focus on development trend and application mode of SOA technology. Some scholars designed and implemented SOA-based distributed video retrieval system [4], adopted standard data model to implement video data retrieval through services-oriented architecture and offered feasible thought for solving media resource decentralize. Sharing isomeric and allopatric audio resource becomes reality. Toda et al. [5] deeply analyzed SOA software engineering development technology and proposed that the introduction of object-oriented software such as component in the development and design of distributed management system could improve system rapidity and reliability and then promote system development efficiency and expansibility. Reyes-Delgado et al. [6] analyzed the adherence degree of four main recognized SDMs and RUP (Rational Unified Process), MSF (Microsoft Solutions Framework), MBASE (Model-Based System Architecting and Software Engineering), and RUP-SOA (Rational Unified Process for Service-oriented 
Architecture) to the best practices of Software Architecture design, and the good application effect was gained.

In fashion design teaching, domestic and overseas scholars widely believe the introduction of multimedia technology has great significance for design teaching. This will be the trend of college teaching improvement. Zhao et al. [7] introduced SOA software algebra model, gave SOA software credibility paradigm and the definition of credibility attribute of SOA software element (service component and service combination). Besides, they proved Markov characteristic of SOA software system under credibility paradigm, proposed comprehensive credibility evaluation model of SOA software system and applied the evaluation model in design teaching. Finally, the good effect was achieved through comparison. However, there are some defects in the application process. For example, the application of multimedia teaching is very widely, including rich teaching resources and videos. Thus, the establishment of video push system has great significance. It is convenient to push the selective teaching video or relevant other videos. Besides, user's demand for a teaching video will change with the teaching content or teaching object. However, the exiting video push system cannot well deal with such change, and it is very hard to complete diversified push services to meet user's needs, which results in network resource waste and chaos [8]. Secondly, current multimedia video teaching is not specially applied in fashion design major. Most teachers teach on the general multimedia equipment. Thus, the teacher needs lots of time to collect professional knowledge. In addition, multimedia data gathered by some teachers are not complete or the knowledge lags behind, which influences the teaching effect [9].

SOA-based simulation and synchronous teaching system designed in this paper owns innovations in two aspects. On one hand, the introduction of SOA can give full play to the function of existing teaching resources, without the need of writing the logic again and improve the effect of multimedia teaching software in integration application. Meanwhile, simulation and synchronous teaching system of clothing template is applied (made by Gelin Brothers Science and Technology Co., Ltd. in Shenzhen, China). The system takes clothing teaching program as the principal line and covers three main courses: product design, industry template, and sewing technology. The system provides lots of vivid teaching courseware, offers courseware teaching and synchronous video teaching for teachers and reduces teacher's repeated workload. On the other hand, multimedia teaching video playing interface push system is added in the distributed software, and it can complete multimedia playing according to interface parameters, with high push efficiency. It can adapt to multiple teaching environments and objects if teaching, integrate rich network resources so as to reach the better teaching effect.

\section{Theoretical construction}

Different from object orientation, SOA takes service as the basic unit of system construction. Each independent logic unit is integrated through deploying SOA. SOA can reduce the difference between the platform and programming language so as to 
achieve cross-platform development. Theory construction of SOA-based distributed teaching software is conducted from three aspects:

\subsection{SOA Implementation Pattern}

For different design demand, SOA owns multiple implementation patterns as follows:

Service agent implementation pattern: in such pattern, the requester does not need to know the details of service provider about the service but gains the service through agent mode. Both parties do not establish the direct connection, as shown in Fig 1.

Packaging service implementation pattern: the pattern can gain SOA system through packing the existing functions into services and solves the integration problem between SOA system and the original system. Packaging can fully embody the advantages of SOA in cross-platform and cross-system, as shown in Fig.2.

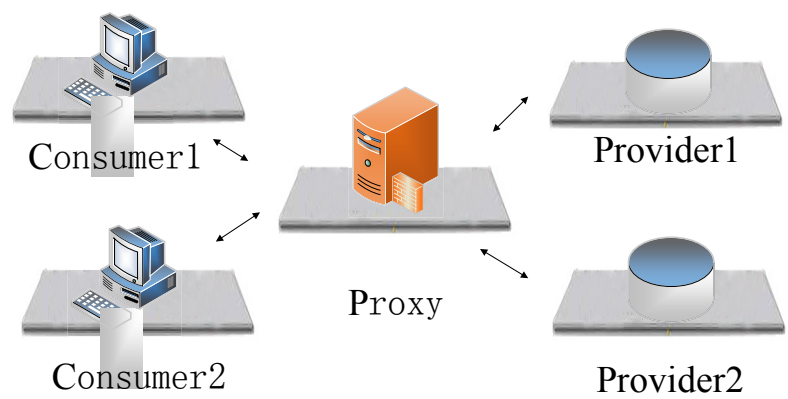

Fig. 1. SOA service agent implementation pattern

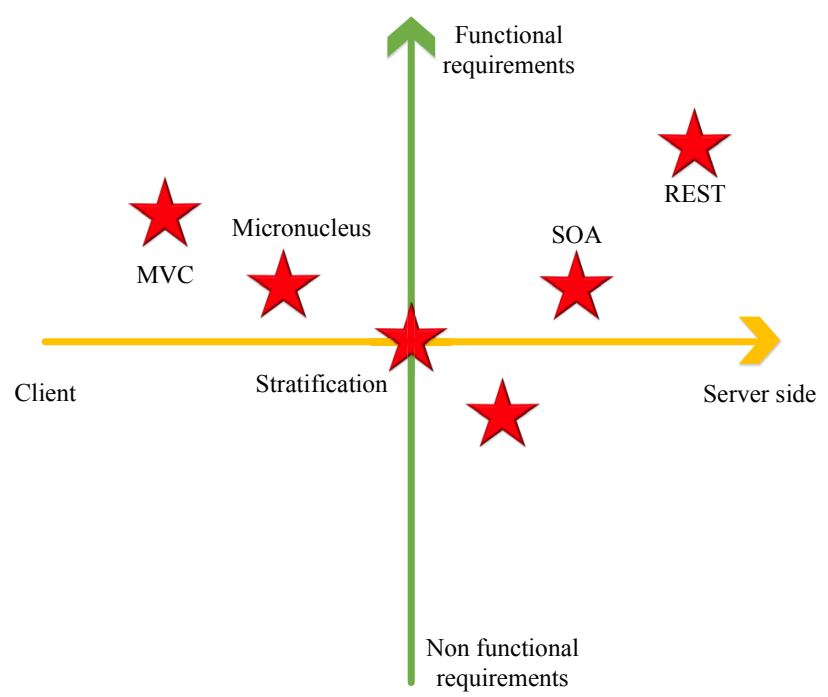

Fig. 2. SOA packaging service implementation pattern 
Virtual service implementation pattern: such pattern combines the above two patterns. The main function is to offer the functions that existing services cannot implement. In other words, the function of existing system is converted to services first through the packaging service pattern, and then the conversion I conducted with the help of a service agent. Finally, Façade [10] mode is used to unify the difference between two services as shown in Fig.3.

Service integration implementation pattern: such mode can fully embody the advantage of SOA in recombination. The brand-new service is gained through recombination of workflow and combining different function demands. Such pattern contributes to improving system efficiency, reducing the pressure of service terminal and gaining the better exhibition effect, as shown in Fig.4.

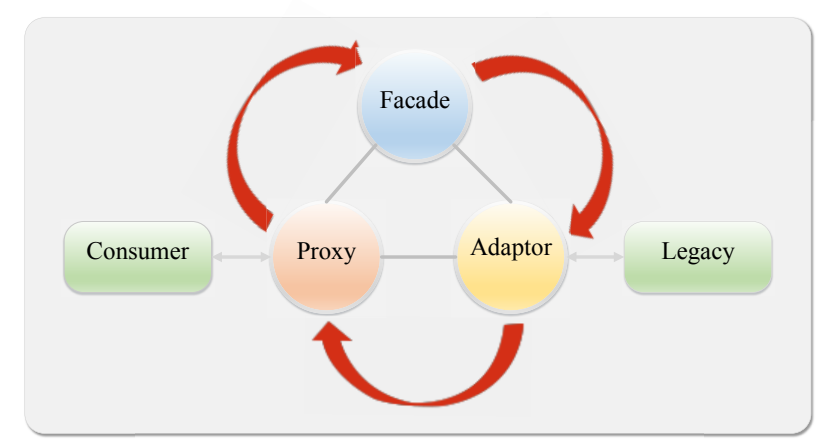

Fig. 3. SOA virtual service implementation pattern

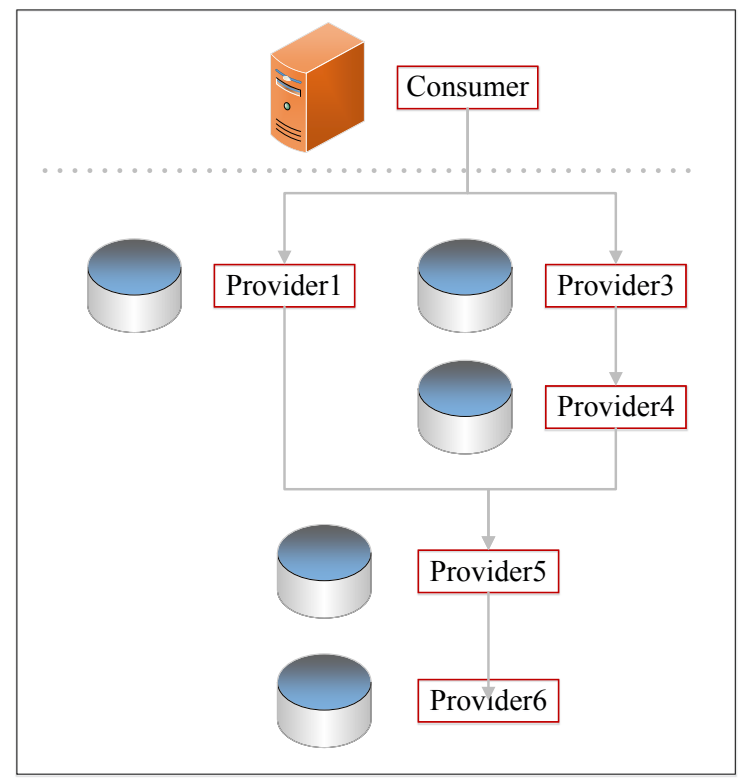

Fig. 4. SOA service integration implementation pattern 
ESB pattern: as a common pattern, ESB-based SOA design pattern can solve service interaction and communication problem. Besides, it supports controllable distributed bottom framework and owns information sharing ability. The interaction methods of ESB mode include synchronous processing and asynchronous processing, as shown in Fig.5.

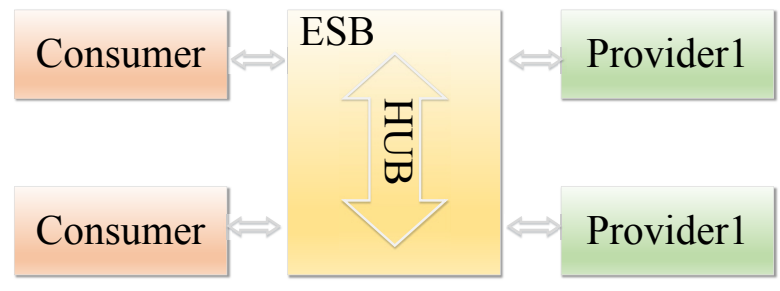

Fig. 5. SOA-based ESB mode

The common SOA patterns include the above, but all patterns are not mutually independent, but supplement each other. A complete SOA development system usually involves the combination of multiple patterns.

\subsection{Distributed System Structure}

The distributed system is the software system which supports distributed processing, i.e. the system which can be operated jointly on the multiprocessor architecture with communication network interconnection [11]. The program of distributed system is distributed on each computer system, including several program modules which can be executed independently. They can be executed at the same time on multiple computers. The distributed system can store system-related data and information in different physical locations in advance, and then connect scattered data by computer network to achieve uniform operation.

\subsection{Development Model Of SOA-Based Simulation and Synchronous Teaching System}

The setup of SOA system development model cannot be separated from service component architecture, business process execution language and service data object as well as the connection among components. The service component architecture can integrate external services to achieve service standardization, self-description and assembly functions. Service data object is used to fast and accurately transmit among each service. The three model components are as follows:

1. Service component architecture (SCA): as the core and essential component, SCA can provide a set of programming model used to construct service-oriented system to thoroughly eliminate the problems of cross-platform. SCA consists of four parts: attribute, service, quote and implementation. 
2. Business Process Execution Language (BPEL): as a kind of programming language written by XML, it can be reflected in automation business process. At present, it is widely applied in the project development related to WEB service. BPEL can combine existing services and define a new WEB service. Meanwhile, BPEL also supports open service standards. BPEL is also called by other system as a WEB service.

3. Service Data Object (SDO): it can provide reliable support for universal application program to make such operations as add, delete, check and modify done easily. The components of SDO include: Data Object, Data Graph, Data Mediator Service and Meta Data.

Based on the above, the connection among different components is required to construct SOA system. So, SOA development mode is shown in Fig.6:

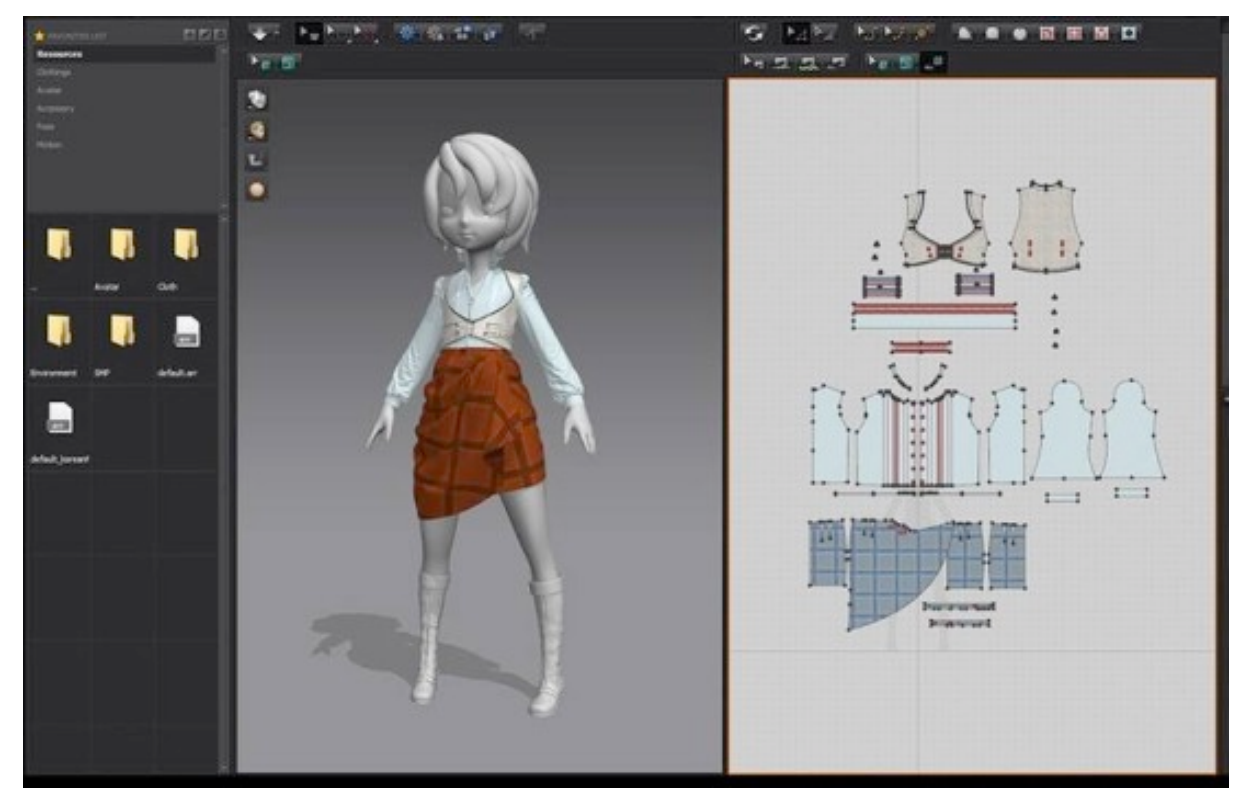

Fig. 6. Multimedia learning platform for Fashion Design based on SOA simulation and synchronous teaching system

As shown in Fig. 6, core processing layer of SOA includes SCA and SDO. It provides consistent interface definition for BPEL through integrating external services. The SOA system development mode is significantly superior to other models in terms of system integration and flexibility and can meet the needs of SOA-based distributed software development. 


\section{Construction of Multimedia Platform for Fashion Design Based on SOA Simulation and Synchronous Teaching System}

\subsection{Design of SOA simulation and synchronous teaching system}

SOA simulation and synchronous teaching system is composed of service provider, service consumer, service register and service binding, as shown in Fig.7.

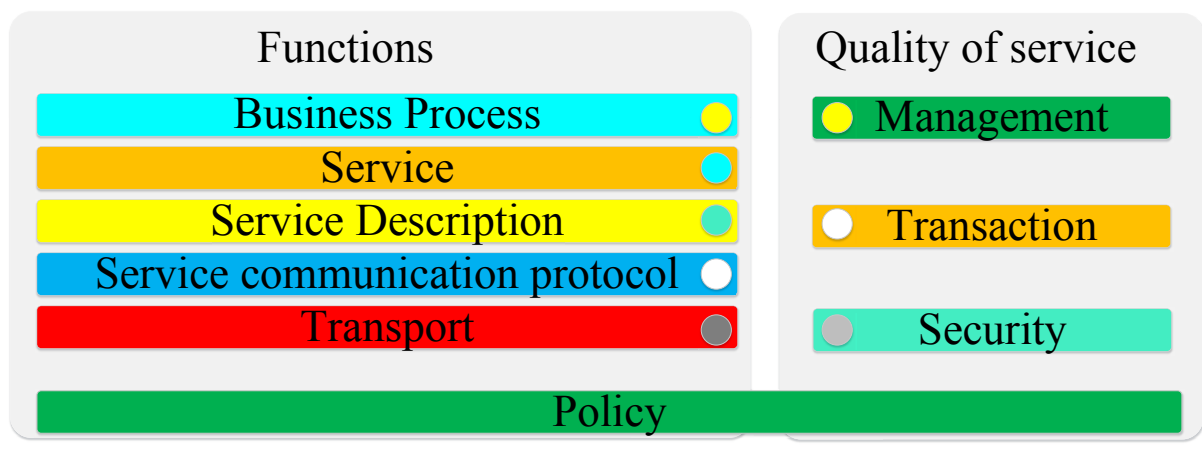

Fig. 7. Structure of Multimedia learning platform based on SOA simulation and synchronous teaching system

The function part includes the following elements: Business Process (users can achieve rearrangement and definition of process through this element), Service (refer to the service called; it can make a response to its own service request), Service Description (including service details), Service communication protocol (method description between service requester and provider), and Transport (public protocol of both parties).

Quality of service part includes the following elements: Policy (it defines a group of rules used to display the information of existing services), Security (it can guarantee system security), Transaction (it refers to the rule which should be followed during completing a common business) and Management (it refers to a group of attributes that both parties manage uniformly).

With regards to the design method, the top-down method is employed to complete design of SOA-based distributed software. Simply speaking, system construction is finished by assignment in steps. Such design method has some important requirements for service division. The function module is continuously simplified through continuous detailing of business process. After service definition is completed, the designer can combine the requirements of Fashion Design multimedia to complete development of SOA-based distributed software so as to satisfy college teaching needs. The interface diagram of SOA-based distributed software is shown in Fig 8. 


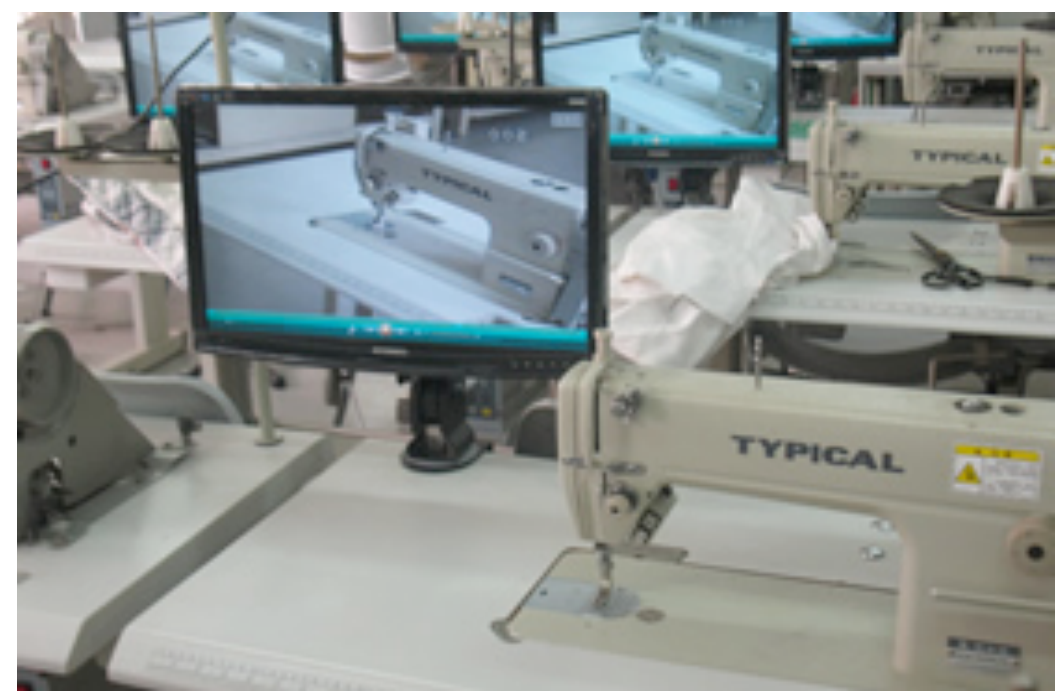

Fig. 8. Application link display of SOA simulation and synchronous teaching system

\subsection{Design of sub-system for multimedia teaching video playing interface push}

SOA simulation and synchronous teaching system introduces a sub-system for multimedia teaching video playing interface push which can make multimedia information act on students according to teaching objectives and features of teaching objects to form rational teaching process structure and reach the optimal teaching effects. With information explosion, multimedia resources are rich. Thus, selecting targeted teaching resources from rich teaching resource videos is of great significance.

Multimedia teaching video playing interface push system includes the following parts:

1. Data input terminal: it is used to complete input of multimedia playing parameters. The multimedia playing list can be automatically generated according to playing parameters. Besides, multimedia playing parameters and the list are sent to interface generation module and push rule base respectively;

2. Interface generation module: it is responsible for receiving multimedia playing parameters at data input terminal and then generating corresponding multimedia playing interface;

3. Push rule base: it receives multimedia playing list of data input terminal and the generates interface push list containing several interface push parameters;

4. Control module: it is equipped with interaction interface, responsible for extracting multimedia playing interface and pushing the multimedia playing interface to multimedia display terminal according to corresponding interface push parameters in the push rule base.

5. Multimedia display terminal: it is used to receive multimedia playing interface and carry out playing operation. 


\subsection{Effect check}

In this paper, the college course of Fashion Style Design in fashion design major was used as the experimental course to test the application effect of SOA-based simulation and synchronous teaching system. The course is composed of two parts: theoretical knowledge of fashion style design, and relevant skill training. The teaching objective is to achieve integration of skills and theoretical knowledge. Classroom teaching of Fashion Style Deign is the key. The teacher needs to teach with multimedia. So, the application of SOA-based simulation and synchronous teaching system in Fashion Style Deign has a good foundation.

Two classes of fashion design major in a University were chosen as the experimental class and control class. Each class included 55 students. Before teaching Fashion Style Deign, the quality level analysis of both classes showed the difference had no statistical significance $(P>0.05)$. The period was 64 . SOA-based simulation and synchronous teaching system was applied for the experimental class. Both the teacher and students might conduct teaching activities with the software and make the best of various functions of the teaching system to promote teaching effect. Traditional multimedia teaching was used for the control class. The teacher mainly introduced features of fashion design through pictures and texts for the control class. The assignments of both classes were same. The final examination was used to test the application effect of SOA-based simulation and synchronous teaching system, including theory test and skill test. After the course ended, both classes were tested. The results of theory test were shown in table 1 and table 2 .

Table 1. Results of theory test

\begin{tabular}{|l|c|c|c|}
\hline \multicolumn{1}{|c|}{ Class } & Average score & The highest score & The lowest score \\
\hline Control group & 85.26 & 98 & 70 \\
\hline Experiment group & 78.97 & 90 & 66 \\
\hline
\end{tabular}

Note: experimental results had statistical significance $(t=5.518, P<0.05)$. There were no students failing in the examination in both classes.

Table 2. Results of skill test

\begin{tabular}{|l|c|c|c|}
\hline \multicolumn{1}{|c|}{ Class } & Average score & The highest score & The lowest score \\
\hline Control group & 82.1 & 99 & 69 \\
\hline Experiment group & 77.37 & 92 & 60 \\
\hline
\end{tabular}

Note: experimental results had statistical significance $(t=3.01, P<0.05)$. There were no students failing in the examination in both classes.

As shown in Table 1 and Table 2, both the theoretical and skill test results of the experimental class improved significantly in the final test. The average, highest and lowest score of the experiment group in the final test are significantly higher than those of the control group. The application of SOA-based simulation and synchronous teaching system in practice gained the obvious effect, and students' scores improved a lot. The results indicate that, SOA-based simulation and synchronous teaching system can reach the expected development objective. 


\section{Conclusions}

Fashion design and other design courses put forward a high requirements for computer aided technology, so SOA-based distributed software development has important significance for multimedia teaching of fashion design. In combination of practical needs of fashion design teaching, SOA-based distributed software was designed and implemented mainly from the perspectives of application and technology in this paper. The application results show that, students' practical ability and theory score improves. The reasons are as follows. Firstly, the teaching system can enhance students' practical abilities, for example, a T-shirt is designed. Different from practical hand painting effect, the advantage of the fashion design style pictures in the teaching system is that the pictures can more vividly express fashion style. Highquality simulated 3D animation can be edited and figured out in a moment, and the picture is closer to the clothes effect. In traditional teaching software, the design is based on experience and feeling rather than formulas. Thus, hand painting ability also improves while the application skills of the software is mastered. This motivates students' enthusiasm, optimizes their operating habits and also promotes their practical performance.

Secondly, the teaching system can accurately take fashion design teaching as the principle line and cover three main courses: product design, industry template, and sewing technology. The system provides lots of vivid teaching courseware and has the function of updating the styles continuously. Meanwhile, it can offer two teaching modes for teachers: courseware teaching and synchronous video teaching, which greatly reduces teacher's repeated workload. Meanwhile, it also promotes students' learning efficiency.

In short, from the perspective of performance, the whole system has reached the expected design objective, and can meet the fashion design teaching requirements. The introduction of multimedia teaching video playing interface push sub-system can offer proper push service for diversified teaching links and teaching objects, effectively improves integration and use ratio of network resource and reaches the higher application effect. The promotion and application of SOA-based distributed software will highlight the advantages of computer aided instruction and offer help for students' employment or further study.

\section{References}

[1] Karande, A.M., Chunekar, V.N., Meshram, B.B. Working of Web Services using SOA. International Journal of Advanced Research in Computer Science, 2010, vol. 1(4), pp. 292296.

[2] Yuan, S.T., Lu, M.R. An value-centric event driven model and architecture: A case study of adaptive complement of SOA for distributed care service delivery. Expert Systems with Applications, 2009, vol. 36(2), pp. 3671-3694. https://doi.org/10.1016/j.eswa.2008.02.024

[3] Nascimento, A.S., Rubira, C.M., Burrows, R., et al. Designing fault-tolerant SOA based on design diversity. Journal of Software Engineering Research \& Development, 2014, vol. 2(1), pp. 1-36. https://doi.org/10.1186/s40411-014-0013-7 
Paper-Multimedia Teaching Platform Construction for Fashion Design Based on Simulation and ..

[4] Li, L., Lu, Q. Design of Distributed Video Retrieval System Based on SOA Technology. Video Engineering, 2014, vol. 38(1), pp. 187-189.

[5] Toda, K. Skill development for SOA, MDA and Trial for Software Production Engineering. Ieice Technical Report, 2008, vol. 107, pp. 7-12.

[6] Reyes-Delgado, P.Y., Mora, M., Duran-Limon, H.A., et al. The strengths and weaknesses of software architecture design in the RUP, MSF, MBASE and RUP-SOA methodologies. Computer Standards \& Interfaces, 2016, vol. 47(C), pp. 24-41. https://doi.org/10.1016/ j.csi.2016.02.005

[7] Zhao, H.Q., Sun, J. A Methodological Study of Evaluating the Dependability of SOA Software System. Chinese Journal of Computers, 2010, vol. 33(11), pp. 2202-2210. https://doi.org/10.3724/SP.J.1016.2010.02202

[8] Paulsson, F., Berglund, M., Arora, A.S. Suggesting an SOA Framework for Modular Virtual Learning Environments: Comparing Two Implementation Approaches. International Journal of Web-Based Learning and Teaching Technologies (IJWLTT), 2008, vol. 3(1), pp. 43-57. https://doi.org/10.4018/jwltt.2008010105

[9] Paik, H.Y., Rabhi, F.A., Benatallah, B., et al. Service Learning and Teaching Foundry: A Virtual SOA/BPM Learning and Teaching Community. 2010, vol. 66, pp. 790-805.

[10] Tere, G.M., Jadhav, B.T. Design Patterns for Successful Service Oriented Architecture Implementation. Bvicams International Journal of Information Technology, 2010, vol. 2(2), pp. 245-250.

[11] Domdouzis, K., Andrews, S., Akhgar, B., et al. Application of a New Service-Oriented Architecture (SOA) Paradigm on the Design of a Crisis Management Distributed System. International Journal of Distributed Systems \& Technologies, 2016, vol. 7(2), pp. 1-17. https://doi.org/10.4018/IJDST.2016040101

\section{Author}

Peng Qu is a Lecturer in the University of Science and Technology Liaoning, Anshan 114051, China.(3121383141@qq.com).

Article submitted 13 February 2018. Resubmitted 18 March 2018. Final acceptance 25 March 2018. Final version published as submitted by the author. 